\title{
Original Series
}

\section{Preceptor Development: Providing Effective Feedback}

\author{
Samaneh T. Wilkinson, MS, PharmD*; Rick Couldry, MS, RPh ; Holly Phillips, PharmD"; \\ and Brian Buck, PharmD, FASHP
}

\begin{abstract}
Feedback plays a significant role in precepting and is indispensable in residency training. As described by the Accreditation Council for Graduate Medical Education, the goal of any postgraduate residency program is to prepare individual trainees to function as qualified practitioners. Although feedback and evaluations have traditionally been synonymous, our goal is to differentiate the two and describe the role of each within resident performance. The goal of this article is to provide preceptors with the tools to provide timely, effective, and quality feedback to residents on a regular basis. Although the focus of this article is on residency training, these concepts can be utilized in student rotations as well.
\end{abstract}

Key Words—evaluation, feedback, preceptor development, resident performance

Hosp Pharm-2013;48(1):26-32

$\mathrm{P}$ roviding feedback effectively, a core function of precepting and a critical step in the learning process, is perhaps the most essential skill for preceptors who are involved in experiential learning. Although feedback seems to be an intrinsic function of precepting, its importance to the success of residents and residency programs is fundamental. Incorporation of formative feedback is necessary to prepare trainees to function as qualified practitioners upon completion of a residency program. Thus, discussions regarding continued improvements in this area are warranted, even in an established preceptor development program.

\section{EVALUATIONS VERSUS FEEDBACK}

Evaluations, a key component to the residency learning process, contain a feedback component but are summative and reflective of overall rotational performance. Typically, evaluation occurs upon completion of an established time frame (week, month) and represents a judgment of how the trainee has performed based on pre-established objectives and goals. ${ }^{1}$ An evaluation may also provide some benchmark assessment. For example, a resident is compared to peers or residents who are at similar points in the program. In an evaluation, there should be no feedback that the resident is hearing for the first time. References to ongoing feedback that was provided during the rotation should be discussed in an evaluation. Unquestionably, the evaluation is an important tool to resident learning and it is a time to reinforce successes or areas for improvement; however, it is not a substitute for ongoing, behavior-based feedback.

\section{FEEDBACK APPROACHES}

There is substantial published information in health care literature regarding the evaluation process but relatively little is published on the art of feedback. Feedback is an ongoing process, a formative one that presents nonjudgmental information that helps the trainee to build on a foundation of skills and behaviors. ${ }^{1}$ It allows the trainee to progress over a time period and work toward a goal. It should be frequent, face-to-face, and provided in small doses., ${ }^{2,3}$ Effective feedback focuses on what was done and the potential consequences

*Assistant Director - Inpatient Clinical Services, PGY-1 Residency Program Director, PGY-2 HSPA Residency Program Coordinator, The University of Kansas Hospital, Kansas City, Kansas; Director - Pharmacy, PGY-2 HSPA Residency Director, The University of Kansas Hospital, Kansas City, Kansas; ${ }^{\ddagger}$ Acute Care Pharmacist Manager, PGY1 Residency Program Director, University of Colorado Hospital, Aurora, Colorado; ${ }^{\S}$ Clinical Associate Professor, University of Georgia, College of Pharmacy, Athens, Georgia. Corresponding author: Samaneh T. Wilkinson, MS, PharmD, Department of Pharmacy, The University of Kansas Hospital, 3901 Rainbow Boulevard, MS4040, Kansas City, KS 66160; phone: 913-588-2300; fax: 913-588-8840; e-mail: swilkinson2@kumc.edu. 
of the action. Feedback assists learners in establishing their goals and critiquing their performance, with the ultimate goal of becoming self-evaluators and motivators. Upon completion of a training program, residents should be able to identify strengths and deficiencies, which will guide future learning throughout their careers. ${ }^{4}$

Performance evaluation is a valuable part of the learning process, but there is a significant distinction between feedback and evaluation. Ende et al has distinguished between the two, explaining that feedback conveys information, whereas the evaluation process confers judgment. ${ }^{3}$ In addition, 4 dimensions of feedback have been identified and described for clinical preceptors in a structured clinical setting. ${ }^{5}$ These dimensions - organization, interaction, impact, and depth - serve as a framework for providing instruction and feedback in the clinical setting. Our recommendations for providing effective feedback will be based on these dimensions and techniques as described by Kogan et al (directive vs elaborative approach). In the directive approach, a preceptor relays observations and judgments regarding the resident's performance in a clinical encounter. The goal is focused primarily on the delivery of information, a "laundry list" of observations. The resident is not actively involved in the feedback discussion. In contrast, the elaborative approach encourages the residents to engage and reflect upon their performance and skill set. There is more dialogue between the preceptor and the resident, with a focus on self-assessment and reflection. The format of the elaborative approach creates an atmosphere of trust and promotes more effective feedback. ${ }^{6}$ Preceptor development programs should actively discuss methods to enhance the elaborative approach to feedback as it can be a more effective process. Hewson et al suggested a set question bank for incorporating the elaborative approach (Table 1). ${ }^{7}$ Development of standard questions for feedback sessions allows the resident to become acclimated to the process and elicits self-assessment. Feedback models, such as the one developed by Hewson et al, suggest a 6-step question-based process to assist in feedback delivery (Table 1). ${ }^{7}$ This model allows the resident to become oriented to the feedback session and elicits self-assessment as well as the development of an improvement plan if necessary.

\section{FACTORS THAT INFLUENCE THE QUALITY OF FEEDBACK}

Several factors potentially impact the process of providing effective feedback. Environmental factors include the frequency, place, and timing of the feedback. Interpersonal factors, including personalities
Table 1. Suggested questions from the Henson feedback model ${ }^{7}$

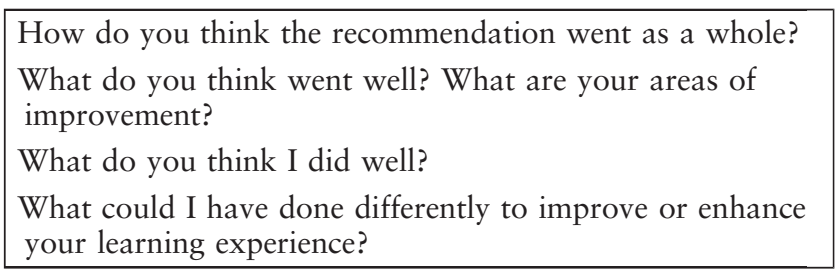

and styles of both the preceptor and resident and the relationship between them, can be barriers if they are not given appropriate consideration. Finally, situational factors, such as the content of the feedback (positive feedback is always easier than constructive feedback), also have an effect.

\section{Environmental Factors}

A neutral, private, setting is always the safest environment for feedback. Residents appreciate oneon-one time and the feeling of importance they get when preceptors take time to talk. This dedicated time establishes rapport between the provider and recipient. Privacy limits the distractions and interruptions that can be inherent in patient care settings.

Ensuring that both preceptor and resident are comfortable with the selected setting for feedback is important. When feedback is constructive and highlights potential areas of improvement for the resident, the importance of a private setting is heightened. Most people will not appreciate even professionally handled and simple "area for improvement" feedback if it is given in a public setting. More serious constructive feedback, such as when someone is wrong, especially if patient care is at risk, should always be handled privately.

Timing of feedback is also critical. If the resident program has the position that feedback should be a daily and regular process, then timing becomes a little easier. Ideally, feedback should be given as chronologically close to the event as possible. Proximity in time helps ensure that both preceptor and resident recall specific details and both are clear on the topic of the feedback. This also helps reinforce actions or behaviors that should be repeated or avoided. Likewise, the close timing of event and feedback makes it easier for residents to ask questions and allows preceptors to make clarifications if needed. Even if preceptors are busy, little real time is often needed to provide effective feedback. Stepping aside from rounds in a quiet hallway for 1 to 2 minutes may be all that is required to meet this need. 


\section{Interpersonal Factors}

The diversity among personality types and communication styles can represent a challenge in providing effective feedback. Individual traits, such as confidence or insecurity, extroverted or shy, factual or sensitive, structured or spontaneous, can affect feedback delivery and reception. There are various learning styles, communication styles, and personality type assessment tools available that can be used to establish a baseline for both preceptors and residents. ${ }^{8}$ One of our go-to tools is Now, Discover Your Strengths $s^{9}$ by Buckingham and Clifton. Future articles in this series will address the utilization of such tools in residency programs. It should be remembered that the responsibility for adapting feedback to best suit the resident falls squarely on the preceptor.

\section{Situational Factors}

Feedback content is possibly the most significant situational factor. In a recent study in the medical literature, the majority of preceptors were found to be comfortable when providing a resident with positive feedback, however when negative feedback was involved, they were less comfortable $(91 \%$ and $64 \%$, respectively). ${ }^{4}$ We have noted that the feedback sandwich (giving positive, negative, and then positive feedback) has been successful when providing difficult feedback; it maintains the trainees' attention and helps them feel more comfortable.

Another situational factor that is often overlooked is the need for specificity in feedback. When performance is good or exceptional, it is easy to comment that the resident did a great job or performed very well. This lack of specificity does not give the learner adequate information. It is more meaningful to link the feedback to a specific action: "You did a great job just now. I really appreciated the way you remained professional and helpful when the physician was clearly upset. You kept calm and asked questions to understand their point of view. Well done!' For constructive criticism, the preceptor should provide an example of how to handle the situation next time. Instead of commenting, "You didn't seem to interact with the team," the preceptor could say, "I would encourage you to take more time and prepare for rounds so that you aren't spending valuable team time pouring over the chart. This allows us as pharmacists to be engaged with much more presence during patient care discussions." By providing specific feedback, preceptors give residents examples to follow in future situations.

\section{Resident Involvement}

Residents also have responsibility in receiving feedback. There are significant differences between the perceptions of residents and preceptors regarding feedback. Medical literature has suggested that residents are often dissatisfied with the quality and amount of feedback received. ${ }^{1}$ Compared with residents, preceptors were more likely to believe that they delivered sufficient amounts of immediate, specific, quality feedback; therefore, engaging the resident in the feedback process is critical. ${ }^{6}$ Given the opportunity to discuss and provide input, individuals will often self-identify areas of improvement. Self-identification can be a crucial component in developing the resident's life-long self-directive learning skills. Openended questions are a useful tool in promoting robust self-evaluation. Questions such as "How do you think that went?" or "What went well/poorly in that situation?" engage the resident and initiate a good feedback discussion. Residents may be fully aware that their performance is lacking, and a simple question can kindly and firmly hold them accountable without engendering bad feelings. A preceptor can follow a simple formula in this situation, making an observation of a behavior or outcome followed by, "Tell me about that." For example, in a situation of tardiness, the preceptor can say, "I noticed you were 15 minutes late this morning. Tell me about that."

One method to engage the resident in the feedback process is to set the tone for the rotation in a prerotation meeting. A review of clear expectations and performance can be incorporated into this meeting. Meeting discussions can include the preferences and style of the preceptor and residents, options for accessibility (paging, phone, text, etc), and specific times for regular formal feedback sessions (Appendix A). This not only demonstrates that the residents' rotation goals have been considered, but also provides needed instructions and guidance for success and, in circumstances where more constructive feedback is needed, serves as a foundation for providing that feedback.

\section{CHALLENGES TO FEEDBACK}

Many challenges exist to providing effective feedback, but the lack of training is the most evident. No formal education for pharmacy preceptors occurs in regard to this skills set. There is a noted disconnect between resident and faculty perceptions in the medical literature, and more evaluation of this should occur in pharmacy residency programs across the nation. Finding an appropriate balance between environment, feedback 
Table 2. Strategies and factors that affect the quality of feedback

\begin{tabular}{ll}
\hline Environment (setting) & - Neutral, private, setting \\
& - Face-to-face \\
\hline Timing & - Away from patient care; minimize interruptions \\
& - Soon after observed behaviors \\
& - Engoing \\
\hline Individualization & - Establish preceptor-resident rapport and relationship \\
& - Encourage self-assessment \\
& - Brainstorm feasible solutions and suggestions for improvement \\
\hline Content & - Consider resident personality and strengths \\
\hline Specificity & - Relevant observed behaviors \\
\hline Preparation & - Spphasize desirable behaviors \\
\hline
\end{tabular}

content, resident personality, and resident-preceptor relationship is necessary for effective feedback.

\section{PRECEPTOR PEARLS: PUT IT INTO PRACTICE}

1. Consider use of a personality assessment tool or communication style tool for residents in order to educate preceptors on adapting feedback to best suit resident learning style. Many tools are available for free on the Internet and take little time to administer. As previously mentioned, our recommendation is Now, Discover Your Strengths.

2. Consider the development of a pre-rotation meeting where mutually agreed upon goals, objectives, and performance evaluation are established (Appendix B).

3. Create a forum where more experienced and talented preceptors can share their knowledge. This can be useful tool early in the residency year. This forum can be as simple as a discussion session where preceptors share examples of feedback sessions that went very poorly and very well. This kind of open forum can foster preceptor-to-preceptor coaching and guidance, which can be rewarding to preceptors and enriching to the program. Another possibility for enhancing feedback skills is to engage residents. Residents can be included in an open discussion session previously described to talk about the traits of feedback that has been most useful to them. This creates a unique situation where the students become teachers and residents are valued as a critical part of the quality of the residency program. Residents might also be included in an open survey asking for examples of feedback that were very good and what was good about them. Compilation of resident feedback on preceptors can also provide useful, individualized information for preceptors on specific areas for improvement on feedback skills.

4. Understand the factors and strategies that affect the quality of feedback (Table 2). Practice incorporating these into weekly feedback sessions to improve preceptor skill set.

\section{DISCUSSION}

Our experience and supporting literature suggest that delivering feedback is a complex process influenced by many factors. Like many skills related to interpersonal communication, some basic principles can be discussed and learned, but the most effective means for skill development is practice. The first step is to make a commitment to provide trainees with informative feedback that is focused on evaluating skills and is provided in a non-judgmental fashion. Effective feedback is critical to the success of a residency program. Feedback is a cornerstone of progress and development for all learners. Ironically, skills in this area can be some of the least taught and most difficult to master; therefore, the inclusion of feedback skills in preceptor development programs is a sound choice for improving the quality of residency programs.

\section{ACKNOWLEDGMENTS}

For Appendices A and B, we thank Marya Warren, PharmD, and Michelle Simonsen, PharmD, pediatric clinical pharmacists at The University of Kansas Hospital.

\section{REFERENCES}

1. Katz PO. Providing feedback. Training Endoscopy. 1995; 5(2):347-555. 
2. Jensen AR, Wright AS, Kim S, Horvath KD, Calhoun KE. Educational feedback in the operating room: a gap between resident and faculty perceptions. Am J Surg. 2012;204(2):248-255.

3. Ende J. Feedback in clinical medical education. J Am Med Assoc. 1983;250:777-781.

4. Sender Liberman A, Liberman M, Steinert Y, McLeod P, Meterissian S. Surgery residents and attending surgeons have different perceptions of feedback. Med Teach. 2005;27(5): 470-472.

5. Frye AW, Hollingsworth MA, Wymer A, Hinds MA. Dimensions of feedback in clinical teaching: a descriptive study. Acad Med. 1996;71(1suppl):S79-81.
6. Kogan JR, Conforti LN, Bernabeo EC, Durning SJ, Hauer KE, Holmboe ES. Faculty staff perceptions of feedback to residents after direct observation of clinical skills. Med Educ. 2012;46:201-215.

7. Hewson MG, Little ML. Giving feedback in medical education: verification of recommended techniques. J Gen Intern Med. 1998;13:111-116.

8. Kolb DA. Experiental Learning: Experience as the Source of Learning and Development. Englewood Cliffs, NJ: Prentice Hall; 1984.

9. Buckingham M, Clifton DO. Now, Discover Your Strengths. New York: Free Press; 2001.

\section{APPENDIX A}

\section{Example of Pre-rotation Syllabus}

\section{PGY-1 Resident Pediatric Rotation}

Rotation Description

The pediatrics rotation is a month-long experiential rotation for pharmacy practice residents, which involves the pharmaceutical management of patients on the pediatric and pediatric intensive care units. The pharmacy resident will work with the pediatric pharmacist to provide support to the multidisciplinary health care team(s). The primary purpose of this rotation is to facilitate familiarity and comfort with the specialty population of pediatrics, including learning about common pediatric disease states and unique dosing considerations (eg, weight-based dosing). The preceptor(s) will serve as a teacher, mentor, resource, and role model for the resident. The resident is expected to be motivated and enthusiastic and to participate completely in the learning process. The resident will take responsibility for his/her learning and progress and will communicate consistently with the preceptor(s) during the rotation regarding the status of learning objectives and support needs. Common pediatric disease states are listed below.

\section{Rotation Expectations}

\section{Self-study responsibilities:}

Residents are expected to complete the reading assignments, understand the material, and prepare student topic discussions $\square$ Obtain resident flash drive and begin reviewing various topics

$\square$ Review the "Considerations in Pediatric Patients"

$\square$ Comprehensive review of the following reading assignments:

$\square$ Meningitis

$\square$ Seizures

$\square$ Asthma

$\square$ Cystic Fibrosis

$\square$ Failure to Thrive

$\square$ Urinary Tract Infections

$\square$ Others as appropriate

\section{Materials}

$\square$ Residents will be provided a flash-drive with learning objectives, required and recommended readings/articles, as well as other helpful information. Flash-drives must be returned to receive completion credit.

$\square$ Residents will provide preceptor a copy of their monthly calendar and will receive a copy of the student calendar for coordination of monthly activities, discussions, exam, and presentation dates.

$\square$ Residents will receive a list of additional references (databases, hospital policies, textbooks, and websites) to which they will have access during the rotation to research unfamiliar medications/disease states

$\square$ Use of electronics including iPads, PALM pilots, and electronic drug database (eg, Lexi-Comp) are helpful but not required. 
Daily monitoring $\&$ profile review of patients' medication orders $\square$ Monitor patients' medication regimens, labs, vitals and clinical progress daily for ways to optimize outcomes

$\square$ Look for opportunities to make clinical interventions, including IV to PO, streamline antibiotics, consolidate therapy, etc.

$\square$ Monitor orders for compliance to pediatric weight-based dosing using appropriate references.

$\square$ Review and process chemotherapy orders, including protocol, tracker, BEACON entry and releasing, IV room coordination.

$\square$ Document pertinent information in medical record (flowsheet, progress notes, IVents, etc).

Direct patient care and interaction responsibilities

$\square$ Perform medication histories and reconciliations on all rounding and off service patients.

$\square$ Identify patient's own medications for use while in the hospital in accordance with KUH policy as needed.

$\square$ Perform discharge counseling to patients and caregivers as needed

Multidisciplinary team interaction $\square$ Attend rounds daily (as applicable); time and location varies greatly, so be on the unit and check with team.

$\square$ Form relationships with physicians, med students, nurses, and other team members.

$\square$ Communicate regularly and frequently with Peds, PICU, Hem/Onc, and off-service residents and physicians regarding patient medication problems, recommendations, and issues.

$\square$ Provide drug information when appropriate to nurses, physicians, students, and team.

$\square$ Follow-up on pharmacy-related questions that arise on rounds.

$\square$ Dress professionally, including white coat (see extern manual for details).

Education

$\square$ Use clinical Resident Rotation Checklist as a guide for personal growth and

learning (w/ preceptor check off).

$\square$ Discuss patient drug therapy problems/questions with preceptor daily.

$\square$ Discuss personal development and clinical problems/questions with preceptor daily.

$\square$ Attend weekly Pediatric Ground Rounds (Fridays at 8:00 a.m. in Wahl Hall West

Auditorium).

$\square$ Research pediatric drug/disease states that are unfamiliar.

Additional activities

$\square$ Complete a personal project as decided upon in collaboration with preceptors.

$\square$ Provide in-service to pharmacy and/or nursing staff.

$\square$ Present twice monthly Journal Club article reviews during the month.

$\square$ Attend Pediatric Ground Rounds (Fridays at 8:00 a.m. in Clendending Auditorium).

Precepting

$\square$ Serve as "co-preceptor" for students. 


\section{APPENDIX B}

\section{Rotation Feedback and Evaluation}

This checklist will be used to assess resident progress and help with grading. It will be used to complete weekly self-assessments/ feedback sessions during the rotation. The instructor reserves the right to make minor adjustments to the list before grading the report. $(1=$ not achieved, $2=$ needs improvement, $3=$ satisfactory progress, $4=$ achieved, NA =not applicable)

\section{Gather, Investigate, and Follow}

_ Able to gather relevant subjective and objective patient information from the patient chart, electronic health record, nurse, medical team, and caregivers pertinent to the disease state.

- Able to identify and use multiple resources for increasing knowledge of disease states, laboratory results, drug dosing, monitoring parameters, and current standard of care.

-Develop your own systematic method to collect, store, and follow patients past medication/medical history, clinical course, and compliance. A patient medication/medical history, clinical course, and compliance should include the following: problem list, list of all prescription, nonprescription, herbals patient has taken during the past month, and compliance history; previously used medications and reasons why they were stopped; allergies, height/weight, hospital admission date, labs, vitals, drug levels.

-Builds rapport with team members and medical staff quickly. Maintains patient privacy and protects the confidentiality of the patients' personal health data.

\section{Assess, Identify, and Analyze}

-Able to analyze and integrate the patient information to draw conclusions, and identify patient-specific, drug-related problems. Able to describe desired therapeutic outcomes and list all therapeutic alternatives that might produce the desired outcomes.

-Able to identify medications that are considered "monitored meds," medications that can be changed from IV to PO, and medications that require renal or hepatic dosing adjustments.

- Able to identify dosing errors (under/overdosing), drug-drug interactions, contraindications, duplicate therapy, duration of therapy completed, adverse effects.

Able to identify medication therapies without indication.

_Able to identify medical indications without treatment.

\section{Plan and Intervene}

-Make recommendations/interventions during rounds at the appropriate time.

- Identify appropriate team member to contact for order clarifications, recommendations, and interventions during nonrounding times.

-When clarifying medication errors, use professional, nonaccusatory language when talking to other team members.

- Obtain order for and identify Patients-Own-Meds (POM) per KUH policy.

_-Provide assistance to physicians for correct order writing. This includes drug name, dose, route, frequency, and mg/kg dosing when needed.

_ Limit the number of verbal or telephone orders written in patient charts unless requested by physician.

-Obtain preceptor co-signature on all orders written in patients' charts.

- Notify team during rounds of changes that will be made according to Pharmacy-to-Dose (PTD) policy.

-Provide team with appropriate information regarding medication policies and procedures.

- Provide team with pertinent drug information including side effects, monitoring parameters, contraindications, and warnings when appropriate.

- Prioritize interventions. Attempt to resolve critical medication-related problems immediately.

- Respond appropriately to resistance by other team members to accept your recommendations.

-Develop a plan for handling obstacles such as difficult team members, limited time, nonformulary, unavailable medications.

\section{Follow-through, Communicate, Document}

-Identify and discuss issues from previous day not clarified with team members. Follow up on unresolved problems from previous night. Prioritize and clarify problems received on the team pager from nurses, physicians, and order entry pharmacists. Respond to questions from physicians and nurses by formulating an accurate, complete, concise response.

- Provide drug information requested verbally and in writing to the team in a timely manner.

- Write orders for drug levels, IV to PO conversions, renal dosing adjustments, and PTD adjustments in patient chart per KUH policy.

Complete a progress note in the patient chart when appropriate for PTD, and POM per KUH policy.

- Notify nurse, physician, or appropriate team members of pharmacy initiated levels or dose changes.Document all medication errors in Patient Safety Net (PSN).Document interventions, communications and significant patient interactions in an IVent.

\section{Resident Signature}

Date 